\title{
MULTICRITERIAL ANALYSIS OF THE ROMANIAN COASTAL ZONE MANAGEMENT
}

\author{
Catalin ANTON, Carmen GASPAROTTI, Eugen RUSU \\ Universitatea Dunarea de Jos Galati, str. Domneasca 47, Galati 80008, Romania \\ corresponding author's email: catanton@gmail.com
}

\begin{abstract}
The coastal zone is a mixed anthropogenic and environmental area (socio-ecological system) characterized by the significant variety of morphological conditions, land uses, which significantly change in time and space.

Natural systems located on the border between the terrestrial environment and the marine environment are characterized by a morphological, ecological and dynamic variety, which changes over short and medium periods of time, under the influence of climatic factors and taking into account from the tidal effect every two hours to wind erosion or wave-related phenomena. In this paper, the authors will choose the optimal approach of strategic management that aims to analyze and evaluate these options by addressing economic, social and environmental factors. The multifactorial approach must consider a careful analysis of stakeholders in a coastal area, having in mind that each area has its peculiarities and needs to be treated separately. When developing the approach for analyzing and evaluating strategic options, the authors will consider the sectorial plans designed for the coastal area, as well as the local, regional and national development plans.
\end{abstract}

Keywords: Romanian coastal zone, Black Sea, economic factors, social and environmental factors

\section{INTRODUCTION}

Integrated coastal management remains, after more than 30 years of history, as the most appropriate way to address current and broad-perspective problems of the areas in question (e.g. Intergovernmental Panel on Climate Change -, 1996, European Commission, 1999). The ICZM is a "governance process and consists of the legal and institutional framework necessary to ensure that the various development plans are integrated with the objectives of protecting the environment and are achieved through the participation of all those affected" [4].

International experience certifies that the benefits of a simultaneous approach to sectorial issues are greater than the implementation of sectorial oriented development plans. ICZM is not intended to replace sectorial specialized management plans, but rather to harmonize and make them complementary. One of the essential dimensions of the ICZM is the integration into existing policies, in public planning and urban planning (territorial planning) policies.

Integrated management, by its integrative nature and aimed at a long-term and medium-term (strategic) perspective, formulates objectives that pursue the progress/development of the region in accordance with the available resources, with a view to diminish restrictive factors and maximize strengths, pursuing the principle of sustainable development, can and must be an essential component in the management of coastal areas.

\section{METHODOLOGY}

\subsection{PESTEL analysis}

Depending on the purpose and scale of planning in coastal areas, there are several tools and methods that can be used in the planning stages. For this work, the authors used several strategic management tools, such as PESTEL analysis, Monte Carlo simulation or calculation of the supportability factor.

In order to effectively analyze the coastal area, the authors have identified the significant activities that take place in this area, activities that have been divided according to their specificity, into activities of an economic nature, social activities and environmental protection activities. The model analyzed is based on an analysis of PESTEL factors (political, economic, sociocultural, technological, environmental and legal). 


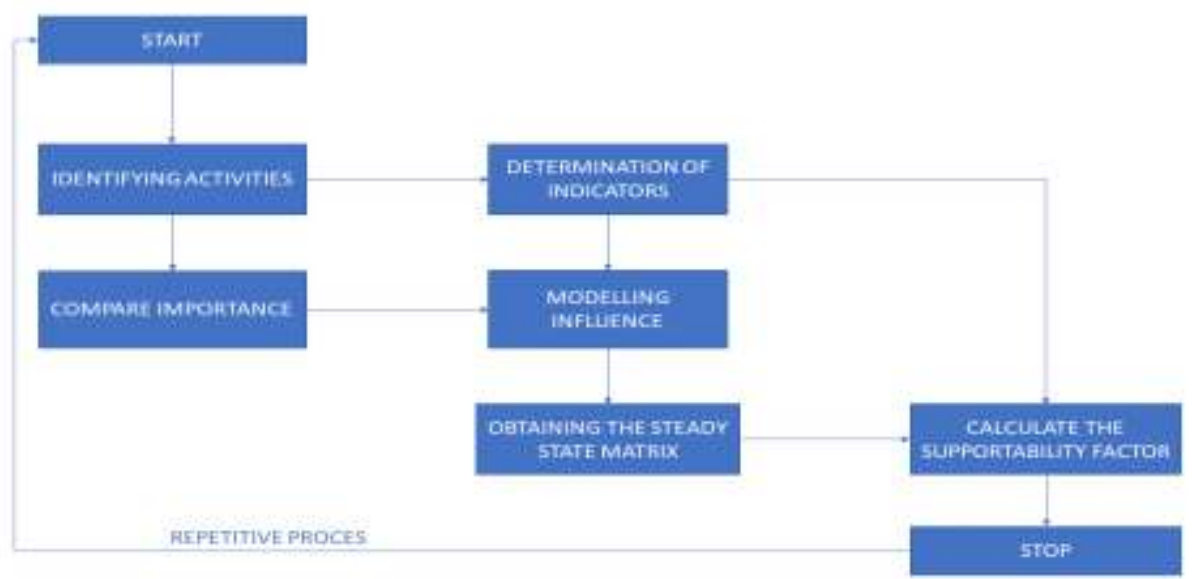

Fig. 1. General framework of the analysis of PESTEL (Political, Economic, Sociocultural, Technological, Environmental Protection and Legislative analysis)

The PESTEL analysis describes a framework of macro-ambient factors used in the environmental scanning component in strategic management. It is part of an external analysis, when a strategic analysis is carried out or a market research is carried out and provides an overview of the various macro-ambient factors to be taken into account. It is a strategic tool for understanding market growth or decline, business position, potential and direction of operations. Figure 1 shows the steps required to perform a PESTEL type of analysis.

\subsection{Use of the Monte Carlo method in} multifactorial analysis of coastal indicators

The multifactorial analysis of the coastal area on the Romanian black sea bank aims to establish the framework within which the activities in the analyzed area take place, to identify pressures that arise as a result of natural or human activities, pressures that can vulnerable the area in the short, medium or long term. What the authors set out to do, instead, is to show which of the activities generate pressure and which activities bear this pressure. For this, the authors turned to a statistical sampling technique to obtain a probabilistic approximation of the solution of a model, like the Monte Carlo simulation. The simulation the authors are talking about combines probability distributions based on existing relationships in models by testing multiple combinations of input variables and storing display results.

The term "Monte-Carlo Method" is used to designate two different techniques. The first technique is to evaluate the defined integrals using random variables. The objective is to calculate,

$$
\int_{a}^{b} F(x) d x
$$

where $\mathrm{x}$ can be a vector, estimating the expression

$$
\int_{a}^{b}[F(x) / p(x)] p(x) d x
$$

where $\mathrm{p}(\mathrm{x})$ is the density function of the random variable defined on $[a, b]$. In this case, the initial problem is converted to that of estimating the mean of $F(x) / p(x)$. One can solve this by generating random values for $\mathrm{p}(\mathrm{x})$ and then calculating the average of $F(x) / p(x)$.

The second meaning of the "Monte-Carlo method" involves replacing a real phenomenon with a statistical experiment, which will be studied using modern computational techniques. The random variables that intervene in the model are generated with the computer by appropriate processes. In order to obtain a correct picture of the evolution of the phenomenon or process studied, the random variables must be estimated with as little deviation as possible from those that occur in reality and the experiment must be repeated a convenient number of times, in order to highlight the main features of the modeled phenomenon.

\subsection{Supportability Analysis}

The mere identification of indicators for the 3 factors proposed for the analysis (economic, social and environmental factors) is not, in the authors' opinion, sufficient to carry out an effective analysis that underpins a model for the Romanian coastal area. By identifying the interaction between factors, one can show the dependence of some of the activities on others, as well as the areas where the interaction of these activities causes conflicts that can lead to the vulnerability of the coastal area (Fig. 2). To see how much the interaction between these indicators of the analyzed factors means, the authors used the concept of a supportability factor. The supportability factor is the framework by which one activity can affect another activity.

For activities that do not interact with each other or interact in an insignificant way, the supportability factor has been set at zero. For activities having a significant or destructive effect on the others, the supportability factor was established as negative, on a scale of zero to minus five, depending on the greater or lesser impact between activities. 


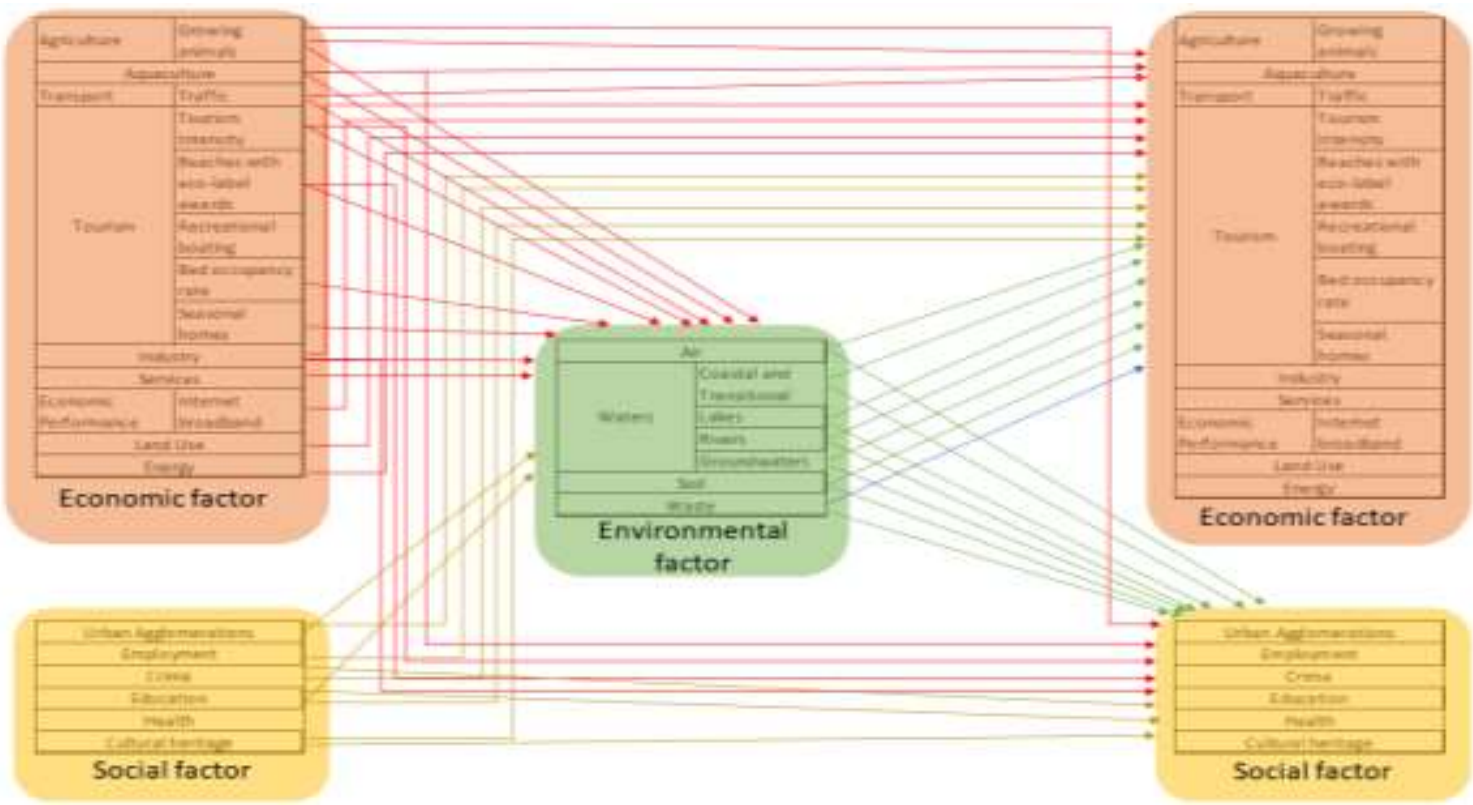

Fig. 2. Interaction between indicators proposed for analysis

Also, if one activity had a positive effect on the other, the supportability factor was also set positively on a scale from zero to five. The impact was established based on existing data from official sources. The allocation of these values was empirical, because of data collected in the area and on similar studies carried out by various researchers or competent authorities in the field.

The calculation of the supportability rate shall be made according to the following formula:

$$
R_{s}=\frac{i\left(e_{p}\right)}{i\left(s_{p}\right)}
$$

where $R_{s}$ indicates the supportability rate, $\mathrm{i}\left(e_{p}\right)$ is the pressure indicator, and $\mathrm{i}\left(\mathrm{s}_{\mathrm{p}}\right)$ is the pressuresupporting indicator.

In the examples below, the authors will also use the calculation of the general support index, according to the following formula:

$$
I_{s g}=\frac{\sum R_{s}}{n * I_{s}},
$$

where $I_{s g}$ represents the general support index, Rs indicates the supportability rate of the indicators analyzed, $n$ represents the total number of indicators and $I_{s}$ represents the indicators analyzed. Similarly, the supportability index can be calculated on each factor.

\section{RESULTS}

The interaction between coastal activities was defined as the generic term land-sea interaction, a term used in the context of marine planning and management in coastal areas. For example, this type of interaction may include, but at the same time, is not limited to the flow of contaminants produced as a result of an agricultural activity from a terrestrial surface to a body of groundwater or surface, which is in contact with coastal waters.

As regarding the quantification of the indicators in the table above, the impact between the indicators can be ascertained by the interaction between the proposed factors (Table 1). Graphically, the table looks like in Fig. 3.

The aggregation of data from different areas of analysis shall be done using measurements corresponding to the calculation rules and standards existing in that area. In the case of insufficient or inconclusive data, aggregations should be treated as approximations of unknown totals or mean values. The aggregation rules are intended to produce estimates for a constant set of data from one period to another and for all indicators. In the analyzed case, the authors used the results of the median ratio from the sum of the interaction values of an analyzed activity and the mean factor values (economic = 0.139053254 , social $=-0.505494505$ and the environment $=0.487179487$ ). The calculation formulas were presented in the previous work.

\section{DISCUSSIONS}

The objective of coastal management is to maximize the benefits of the coastal area and to minimize conflicts and the harmful effects of some activities on others.

This paper aims to find a management model that, on the one hand, can be applied to the peculiarities of the Romanian area and, on the other hand, can be applied to other coastal areas. The analysis started from the peculiarities of the analyzed area, using a matrix of economic, social and environmental factors, to which other factors of the PESTEL analysis (i.e. political, legislative and technological factors) can be added. 
Table 1. Assessing the impact of coastal activities

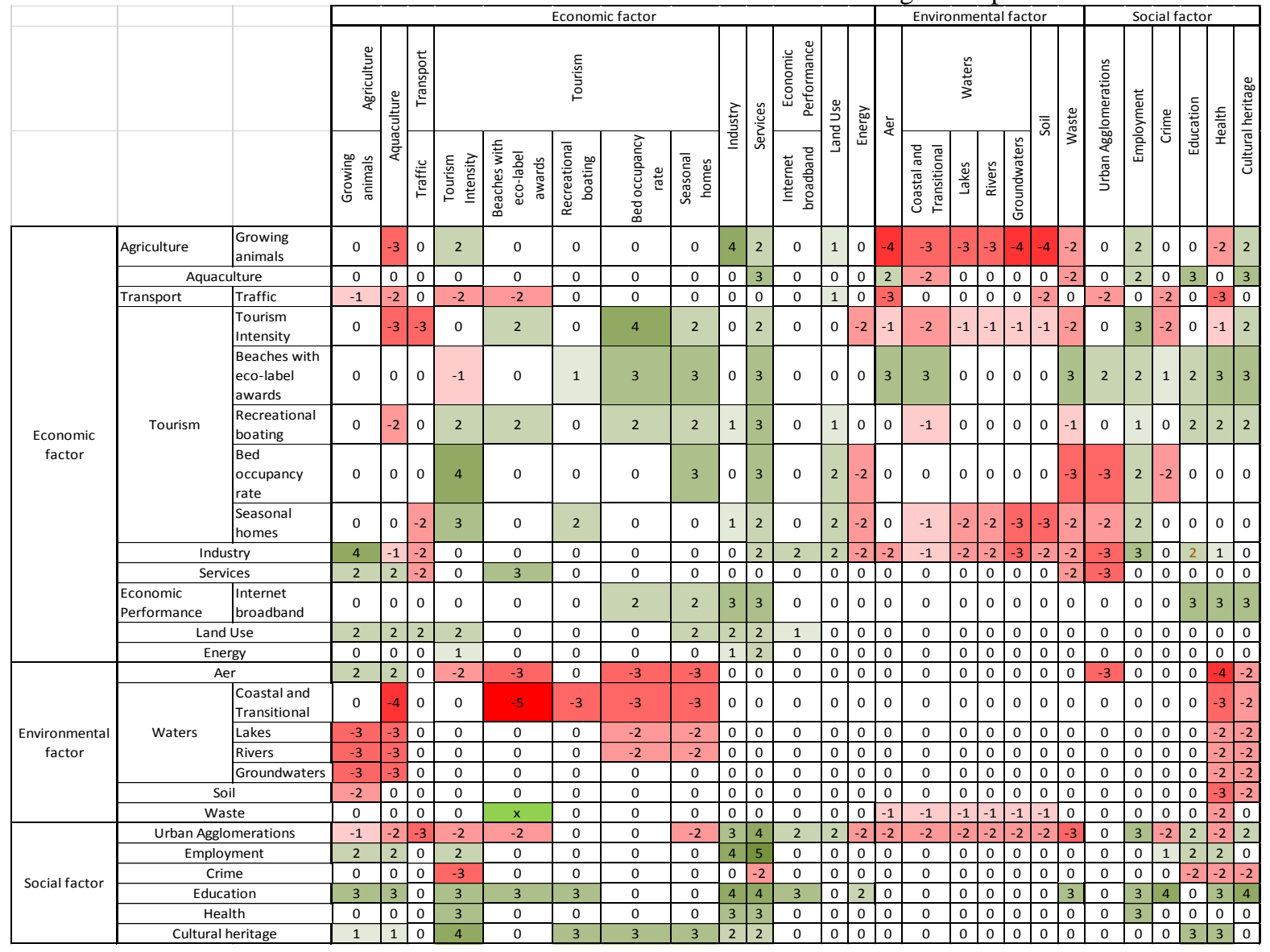

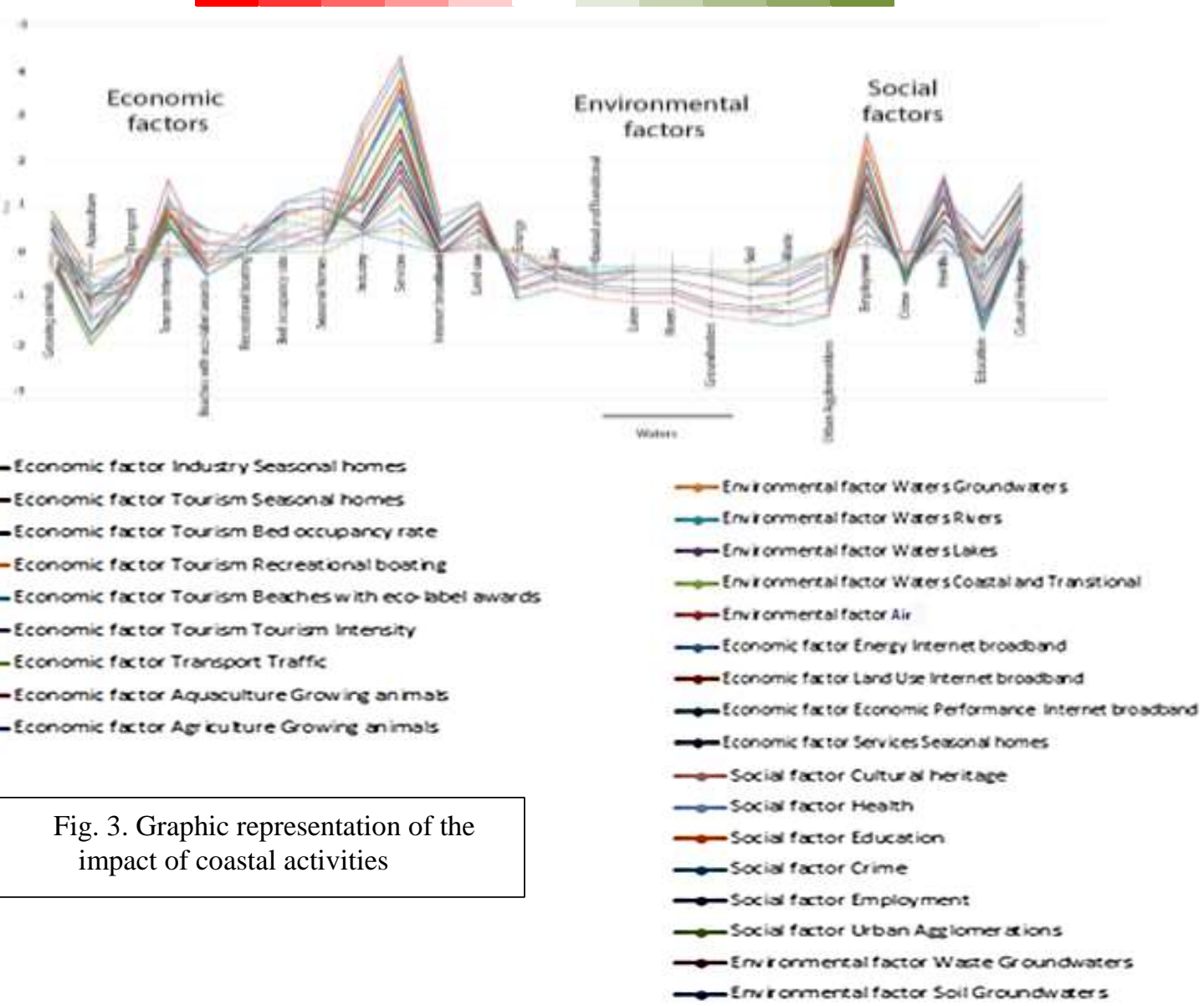


It is necessary to validate the new model over time. Periodically, with the help of a multi-criterial analysis, the validation of the model will show possible changes in the analyzed factors. Local, regional, national and international plans and strategies should be integrated into the analysis. Using a holistic perspective, the model avoids traditional sectorial interconnections between coastal systems and uses integrated management approaches.

As mentioned above, when determining the supportability factor, it was established that, if the activities were not affected by each other or were insignificant, they would have a zero value. Also, depending on the exerted pressures, the value of the activity indicator can have a positive or negative impact and the value will be between minus five and five. If the impact of one activity on the other activity is negative, it means that a pressure affects an activity. A set value of -5 is the maximum, within which an activity can withstand the pressures of other activities. Above this value, the activity can no longer exist in that area. For example, excessive tourist activity can lead to habitat destruction and, by implication, the disappearance of species from the protected area. In this case, the interaction between excessive tourism and the environmental factor (soil, water) can be -5 .

The allocation of the value scale, between -5 and +5 , is not random; it is mainly based on the theory of risk analysis. A risk assessment consists, in this case, in studying the likelihood that the activity will reach a satisfactory/unsatisfactory performance. In this context, the probability should be interpreted as an index where a value of +5 represents a complete certainty that a positive prediction will be confirmed, a value of -5 represents the certainty that a negative prediction will be confirmed and intermediate values can be identified for any situation between the two extremes. The probability tool was used as an assessment tool. Values of this scale may be generated by the experience of specialists in the field, who analyze these situations. Probability thresholds reflect the perception of decision-makers about the uncertainty with which identified risks may be associated. In some of the analyzed activities, a qualitative assessment is required, while a quantitative assessment is required in others.

This theory was initiated by a Monte Carlo analysis, developed in the 1940s, and it is a computerized method that uses statistical sampling techniques to obtain a probabilistic approximation with the solution of a model.

\section{CONCLUSIONS}

This paper is part of a broader research on the impact of coastal activities in the conditions of sustainable development, which will be completed by the doctoral thesis of the first author. The strategic analysis of the Romanian Area on the Black Sea coast has identified a model, in which existing activities, as well as pressures arising from the natural or anthropogenic factors of these activities, can make the area under review vulnerable in short, medium and long term. The multi-criterial analysis model used in the coastal area of Romanian coastal zone identified the relevant forces that exerted pressure on this coastal and marine area. Improving the quality of environmental factors, groundwater quality, is a clear objective for the authorities, in the medium and long term. Furthermore, the diversification of economic activities and the identification of new economic activities in the area under review will provide an aspect of sustainability. However, in order to develop this area, the degradation of coastal ecosystems must be mitigated, providing a common framework for managing multisectorial activities and maintaining options for future uses of coastal resources. The application of this model will create a multisectorial perspective and approach that considers all the interests of sectorial and stakeholders and addresses economic, social, environmental and environmental issues.

Quantifying these activities using the supportability factor makes even clearer how much pressure is on one or another and where this pressure comes from.

It also should be made clear that, for a model to be as viable as possible, the quality of the collected data, as well as the timeliness of such data, are very important. The use of data from different periods can lead to the development of different models. Also, the calibration of the model can be done over time by constantly updating the entered data.

\section{ACKNOWLEDGMENTS}

This work is supported by the project ANTREPRENORDOC, in the framework of Human Resources Development Operational Programme 2014-2020, financed from the European Social Fund under the contract number 36355/23.05.2019 HRD OP /380/6/13 - SMIS Code: 123847."

\section{REFERENCES}

1. Iticescu C., Georgescu L. P., Ţopa C. M., Murariu G. (2014) Monitoring Danube water quality near Galati City, Journal of Environmental Protection and Ecology, 15(1), pp. 30-38.

2. Iticescu C., Murariu G., Georgescu L. P., Burada A., Tुopa C. M. (2016) Seasonal variation of the physico-chemical parameters and Water Quality Index (WQI) of Danube water in the transborder Lower Danube area, Revista de Chimie (Bucharest), 67(9), pp. 1843-1849.

3. Iticescu C., Georgescu L. P., Gurau G., Murarescu M., Dima D., Murariu G., Gheorghies C. (2015) Methods to reduce environmental impact of muni- 
cipal waste water sewage sludge, Environmental Engineering and Management Journal, 14(10), pp. 2457-2463.

4. Study ICZM for the Project "Improvement of the Integrated Coastal Zone Management in the Black Sea Region”, 2014

5. Rusu L.; Butunoiu, D.; Rusu E. (2014) Analysis of the extreme storm events in the Black Sea considering the results of a ten-year wave hindcast, Journal of Environmental Protection and Ecology, 15(2), pp. 445-454.

6. Zanopol A. T., Onea F., Rusu E. (2014) Evaluation of the coastal influence of a generic wave farm operating in the Romanian nearshore, Journal of Environmental Protection and Ecology, 15(2), pp. 597-605.

7. Panaitescu M., Panaitescu F. V., Anton I. A., Anton C. (2016) A Method for flow modelling of vulnerable areas, Journal of Marine Technology and Environment, 2, pp 43-48, Constanta, Romania.

8. Domnisoru L., Modiga A., Gasparotti C. (2016) Global strength assessment in oblique waves of a large gas carrier ship, based on a non-linear iterative method, ModTech International Conference Modern Technologies in Industrial Engineering IV, IOP Conference Series: Materials Science and Engineering, 145, pp. 1-8, 082009, doi:10.1088/1757-899X/145/8/082009.

9. Gasparotti C., Rusu E. (2016) A rewiew concerning the rogue waves and their impact on navigation conditions, Mechanical Testing and Diagnosis, 6 (3), 10, Galati, Romania

10. Rusu E. (2016) Reliability and applications of the numerical wave predictions in the Black Sea, Frontiers in Marine Science, 3, p. 95.

11. Makris C., Galiatsatou P., Tolika, K. et al. (2016) Climate change effects on the marine characteristics of the Aegean and Ionian Seas, Ocean Dynamics, 66(12), pp. 1603-1635.

12. Anton C., Gasparotti C., Raileanu A., Rusu E. (2017) Towards integrated management and planning in the Romanian Black Sea coastal zones, $A U D C$, 13(5), pp. 59-71, Galati, Romania.

13. Anton C., Rusu E., Mateescu R. (2017) An analysis of the coastal risks in Romanian nearshore, Mechanical Testing and Diagnosis, 7(1), 18, Galati, Romania

14. Popa P., Timofti M., Voiculescu M., Dragan S., Trif C., Georgescu L. (2012) Study of physicochemical characteristics of wastewater in an urban agglomeration in Romania, Scientific World Journal, 549028, doi: 10.1100/2012/549028.

15. Iticescu C., Georgescu L. P., Murariu G., Topa C., Timofti M., Pintilie V., Arseni M. (2019) Lower Danube water quality quantified through WQI and multivariate analysis, Water, 11(6), 1305, doi:10.3390/w11061305. 